\title{
Depth dependence of defect density and stress in GaN grown on SiC
}

\author{
N. Faleev ${ }^{\text {a) }}$ \\ Department of Electrical and Computer Engineering, University of Delaware, 140 Evans Hall, Newark, \\ Delaware 19716 \\ H. Temkin \\ Department of Electrical Engineering, Texas Tech University, Lubbock, Texas 79409
}

I. Ahmad and M. Holtz

Department of Physics, Texas Tech University, Lubbock, Texas 79409

Yu. Melnik ${ }^{\text {b) }}$

TDI Inc., 12241 Plum Orchard Drive, Silver Spring, Maryland 20904

(Received 13 July 2005; accepted 1 November 2005; published online 21 December 2005)

We report high resolution x-ray diffraction studies of the relaxation of elastic strain in GaN grown on $\mathrm{SiC}(0001)$. The $\mathrm{GaN}$ layers were grown with thickness ranging from 0.29 to $30 \mu \mathrm{m}$. High level of residual elastic strain was found in thin (0.29 to $0.73 \mu \mathrm{m}$ thick) $\mathrm{GaN}$ layers. This correlates with low density of threading screw dislocations of $1-2 \times 10^{7} \mathrm{~cm}^{-2}$, observed in a surface layer formed over a defective nucleation layer. Stress was found to be very close to what is expected from thermal expansion mismatch between the $\mathrm{GaN}$ and $\mathrm{SiC}$. A model based on generation and diffusion of point defects accounts for these observations. (C) 2005 American Institute of Physics.

[DOI: $10.1063 / 1.2141651]$

\section{INTRODUCTION}

Growth of gallium nitride has been intensively studied due to important limitations in achieving high crystal quality in epitaxial material. Much of the impetus for this research comes from applications of the wide-band-gap $\mathrm{GaN}$ and $\mathrm{Al}_{x} \mathrm{Ga}_{1-x} \mathrm{~N}^{1-3}$ Light emitting diodes and field effect transistors have been produced using $\mathrm{GaN}$, with good performance, despite the poor crystal quality available relative to other electronic materials. The primary limitation on crystal quality is the need to grow $\mathrm{GaN}$ on foreign substrates $\alpha-\mathrm{Al}_{2} \mathrm{O}_{3}$ (sapphire) ${ }^{1}$ and $6 \mathrm{H}-\mathrm{SiC},{ }^{4}$ as well as silicon. ${ }^{5}$ The lattice constant mismatches between $\mathrm{GaN}$ and substrates sapphire and $\mathrm{SiC}$ are -13 and $+4 \%$, respectively. ${ }^{6}$ These values are prohibitively large, making epitaxial growth difficult. Since growth is always performed at elevated temperatures, differences between the thermal expansion coefficients of $\mathrm{GaN}$ and either substrate material induce further stress upon cooling to room temperature. These stresses are compressive and tensile for $\mathrm{GaN}$ grown on sapphire and $\mathrm{SiC}$, respectively, and can reach $\sim 1 \mathrm{GPa}$ in magnitude.

Crystalline defects originate as point defects at the growing surface. The primary causes for point defect generation are strain at the growth temperature and/or imperfect stoichiometry at the growth surface. Relaxation begins with singleaxis waviness or corrugation of crystalline planes, ${ }^{7,8}$ continues by increased roughness of the surface, ${ }^{9}$ and appearance of growth nucleus on the growth surface. ${ }^{10}$ The first stage of this process ends with the creation of point defects on the surface, ${ }^{11,12}$ due to a coalescence of nucleus. The process of

\footnotetext{
${ }^{\text {a) }}$ Author to whom all correspondence should be addressed; electronic mail: faleev@mail.eecis.udel.edu

${ }^{b)}$ Present address: OmniGuide Communications, Inc., One Kendall Square Bld. 100, Cambridge, MA 02139
}

diffusion of point defects follows along with spatial redistribution and structural transformation of point defects by the creation of point defect clusters, stacking faults, dislocations, and dislocation accumulations (dislocation walls). In IIInitride epitaxial layers, threading dislocations grow from the first interface and extend to the surface. ${ }^{10,13}$ Various growth procedures have been developed to reduce the impact of high lattice mismatch and defects on growth conditions and device fabrication. These procedures include the use of buffer layers to seed growth of the epitaxial layers ${ }^{5}$ and lateral epitaxial overgrowth. ${ }^{14}$ Buffer layers affect a transition from heteroepitaxy to a growth mode akin to homoepitaxy. To date, the lowest reported threading dislocation density in thin $(260 \mathrm{~nm})$ nitride layers is about $2 \times 10^{5} / \mathrm{cm}^{2}$ in AlN thin layer grown by molecular beam epitaxy (MBE) on Sapphire (0001) substrate, ${ }^{15}$ and is about $2 \times 10^{7} / \mathrm{cm}^{2}$ in GaN layer grown by hydride vapor phase epitaxy (HVPE) on $\mathrm{SiC}(0001) .{ }^{16}$

Despite a number of studies addressing stress in epitaxial $\mathrm{GaN},{ }^{17}$ the dependence of defect generation, type, and diffusion on epilayer thickness and distance from the epitaxial layer-substrate interface is still not completely understood. In this article, we report high resolution x-ray diffraction (XRD) studies of the defect morphology in GaN grown epitaxially on $6 \mathrm{H}-\mathrm{SiC}(0001)$ substrates. Detailed reciprocalspace mapping (RSM), using several Bragg diffraction reflections, provides us with information about misfit and threading dislocation densities for epilayers over a broad range of thicknesses. We observe a pronounced dependence of threading dislocation densities on thickness, exhibiting low levels at $\sim 0.3-0.6 \mu \mathrm{m}$ from the substrate. We directly compare these results with Raman stress measurements.

The remainder of this article is organized as follows. Following a description of the growth, XRD, and Raman 
experiments, we discuss the dependence of defect density and residual elastic stress on layer thickness. We then describe the RSM measurements for depth profiling the defect properties. Finally, we discuss and interpret our results in terms of point defects generation and their structural transformation during epitaxial growth, and implications for growth experiments.

\section{EXPERIMENTAL METHODS}

Single layer $\mathrm{GaN} / \mathrm{SiC}(0001)$ was grown using hydride vapor phase epitaxy (HVPE). The HVPE processes were performed in a horizontal open-flow reactor under the same conditions ${ }^{18}$ at growth temperature $1020^{\circ} \mathrm{C}$. An initial GaN layer $(25-30 \mathrm{~nm})$ was grown to best accommodate the lattice mismatch in the initial stage of epitaxial growth. ${ }^{19}$ No AlN buffer layer was used. The main epitaxial layers were grown immediately after the initial GaN layer is established. The total thickness of $\mathrm{GaN}$ layers varies over a large range from $290 \mathrm{~nm}$ up to $\sim 31 \mu \mathrm{m}$. The growth rate was $300-400 \mathrm{~nm} / \mathrm{min}$.

X-ray diffraction studies were performed using an X'Pert MRD (Philips) high-resolution diffractometer in double- and triple-axis alignment with $\mathrm{CuK}_{\alpha 1}$ monochromatic radiation. A four-bounce Bartels $\mathrm{Ge}(220)$ crystal monochromator and a three-bounce $\mathrm{Ge}(220)$ analyzer crystal were used for high-resolution measurements. The analyzer separates the coherent and diffuse scattered radiation, thus permitting measurement of the coherent and diffused components of the scattered radiation and their spatial distribution by reciprocal space maps (RSM). The triple-crystal $2 \theta-\omega$ and $\omega \mathrm{x}$-ray diffraction curves will be referred to here as $\mathrm{x}$-ray rocking curves (RCs). An absolute measurement of lattice parameters $a$ and $c$ have been done by triple-crystal method described in Ref. 20. Both symmetrical (0002), (0004), and (0006) and asymmetrical (11.4) and (10.5) reflections have been used for precise determination of corresponding $2 \theta$ Bragg angles and calculation of lattice distances. The difference between our measured lattice constants and previously reported parameters for strain-free GaN (Ref. 21) were used to evaluate the elastic strain in our samples.

Micro-Raman measurements were conducted with the samples at room temperature. Excitation was 488 -nm light from an argon ion laser. Scattered light was filtered using a holographic notch filter, dispersed using a single-grating spectrometer with $0.5-\mathrm{m}$ focal length, and detected using a liquid-nitrogen cooled, charge-coupled device array. Calibration of the multichannel system is accomplished using laser plasma lines and calibrated lamps. Spectroscopic resolution for this system is $\sim 2.5 \mathrm{~cm}^{-1}$; Raman energy shifts of $0.1 \mathrm{~cm}^{-1}$ are reliably detected. ${ }^{22}$

\section{A. Studies of layers with varying thickness}

Figure 1 shows a $2 \theta-\omega$ coherent rocking curve for $\mathrm{GaN}$ layers with thickness $0.29 \mu \mathrm{m}$ (a) and $0.73 \mu \mathrm{m}$ (b). For the $0.29 \mu \mathrm{m}$ layer in Fig. 1(a), the full width at half maximum (FWHM) and the period of sharp interference fringes observed were both $\sim 55$ arcsec and coincide with the calculated curve for an ideal GaN layer with constant lattice $c$ and
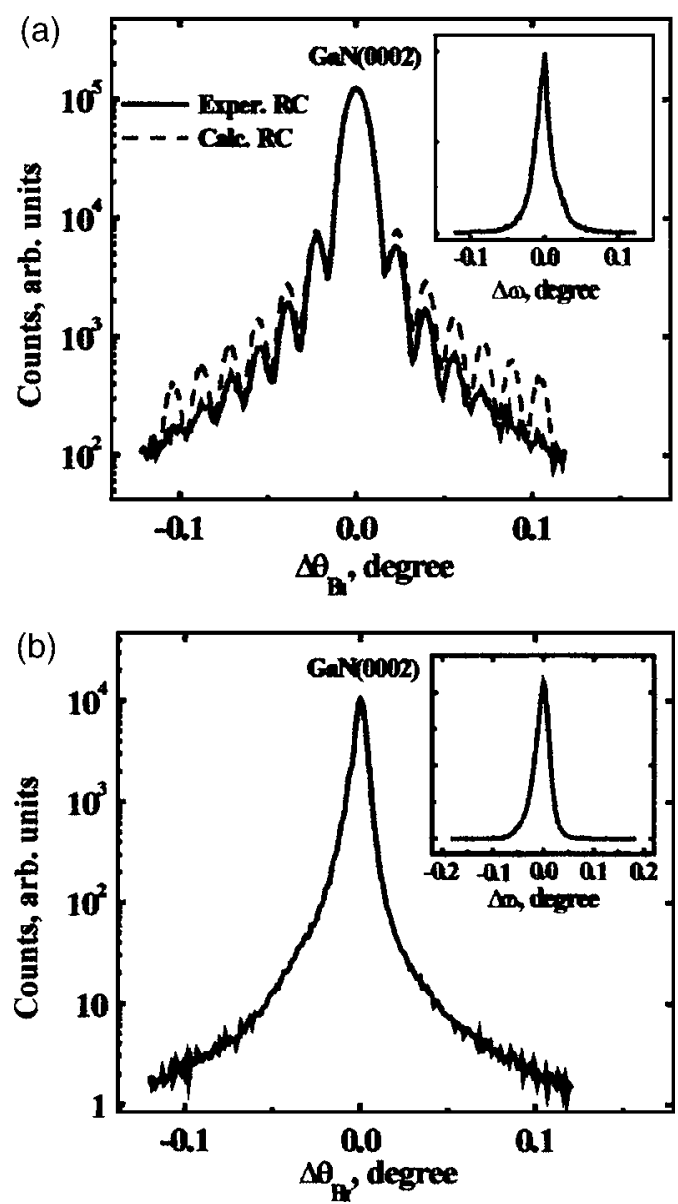

FIG. 1. (a) Experimental and calculated $2 \theta-\omega$ Rocking Curves in the vicinity of $\mathrm{GaN}(0002)$ reflection. Sample 1, $290 \mathrm{~nm}$ thick $\mathrm{GaN}$ layer on $\mathrm{SiC}(0001)$ substrate. Inset shows $\omega \mathrm{RC}$ measured with analyzer crystal installed on the maximum peak position of $2 \theta-\omega$ diffraction curve. (b) Experimental $2 \theta-\omega$ rocking curve measured in the vicinity of $\mathrm{GaN}(0002)$ reflection. Sample 5, more then $730 \mathrm{~nm}$ thick GaN layer on $\mathrm{SiC}(0001)$ substrate. Inset shows $\omega \mathrm{RC}$ measured with analyzer crystal installed on the maximum peak position of $2 \theta-\omega$ diffraction curve.

sharp interfaces. This indicates a consistently strained layer with negligible gradient to the lattice parameters along the growth axis. Fringe intensities in Figs. 1(a) noticeably differ due to domain misorientation in the layer. Macroscopic nonhomogeneity of the thickness of this layer, evaluated from the full angular range of the x-ray interference pattern, is about $30 \mathrm{~nm}$. Our results are consistent with Ref. 23, although they did not address questions of crystal perfection.

Coherent $\omega \mathrm{RC}$ for the $0.29-\mu \mathrm{m}$ thick sample is shown the inset of Fig. 1(a). The FWHM of this curve is 75 arcsec. Based on this, the average lateral coherence length of the crystalline domains is $\sim 720 \mathrm{~nm}$. The corresponding average density of threading screw dislocations is $2.0 \times 10^{7} / \mathrm{cm}^{2}$. The shape of the $\omega$ peak [Fig. 1(a) inset], with wide base and narrow top, is typical for epitaxial layers in which the crystalline perfection drastically changes from the substrateepilayer interface to the surface (see Ref. 24). The wide base of the $\omega \mathrm{RC}$ is related to incoherent diffuse scattering stemming from crystalline disorder, which we attribute to the initial intermediate growth region. The density of threading dislocations in this part of the layer exceeds $1 \times 10^{10} / \mathrm{cm}^{2}$. ${ }^{10} \mathrm{In}$ 
TABLE I. Sample designations, GaN thickness, and results of the XRD studies.

\begin{tabular}{ccccccc}
\hline \hline Sample & $\begin{array}{c}T_{\text {Layer }} \\
(\mu \mathrm{m})\end{array}$ & $\begin{array}{c}2 \theta-\omega \text { RCs } \\
\text { FWHM } \\
(\operatorname{arcs})\end{array}$ & $\begin{array}{c}D_{\text {Coh } \perp}(\mathrm{nm}) \\
1\end{array}$ & $\begin{array}{c}\omega \text { RCs } \\
\text { FWHM } \\
(\operatorname{arcs})\end{array}$ & $\begin{array}{c}D_{\text {Coh } \|} \\
(\mathrm{nm})\end{array}$ & $\begin{array}{c}\text { Screw } \\
\text { dislocations } \\
\left(\mathrm{cm}^{-2}\right)\end{array}$ \\
\hline 2 & 0.29 & 55 & 310 & $70-80$ & $650-750$ & $2.0 \times 10^{7}$ \\
3 & 0.42 & 38 & 435 & 55 & 970 & $1.0 \times 10^{7}$ \\
4 & 0.58 & 29 & 575 & 120 & 445 & $4.8 \times 10^{7}$ \\
5 & $\geqslant 0.68$ & 24 & 685 & 145 & 370 & $6.8 \times 10^{7}$ \\
6 & $\geqslant 0.73$ & 23 & 730 & 165 & 325 & $8.9 \times 10^{7}$ \\
7 & $15 \mu \mathrm{m}$ & 32 & 530 & 430 & 125 & $6.2 \times 10^{8}$ \\
8 & $20 \mu \mathrm{m}$ & 32 & 520 & 385 & 140 & $4.9 \times 10^{8}$ \\
9 & $25 \mu \mathrm{m}$ & 27.5 & 605 & 360 & 150 & $4.3 \times 10^{8}$ \\
\hline \hline
\end{tabular}

the near-surface main layer, this density drops by nearly three orders of magnitude resulting in the more intense and narrows coherent part of the $\omega \mathrm{RC}$.

Figure 1(b) shows the same measurements for the $0.73 \mu \mathrm{m} \mathrm{GaN}$ layer. Here the FWHM diminished to 23 arcsec, as expected for the thicker layer. The slight asymmetry seen in the $2 \theta-\omega \mathrm{RC}$ indicates the onset of elastic strain relaxation in thicker layers. Interference fringes are completely extinguished due to the appearance of edge dislocations in the volume of the epitaxial layer and deterioration of vertical coherence of the layer. The inset to Fig. 1(b) shows the $\omega$ RC peak to be 165 arcsec broad, from which we obtain lateral coherence length $325 \mathrm{~nm}$ and density of threading screw dislocations $9 \times 10^{7} / \mathrm{cm}^{2}$. The shape of the $\omega$ RC peak in the inset to Fig. 1(b) is more symmetric than the corresponding inset in Fig. 1(a) due to the greater layer thickness. The broad base of the $\omega$ RC is not clearly seen, as in the inset to Fig. 1(a). We conclude that the highly disordered interface sublayer remains very thin in comparison to the total thickness, analogous to the $0.29 \mu$ m layer. ${ }^{10}$

Table I summarizes the main parameters from the x-ray measurements for each sample studied. There are several important points regarding Table I. First, the vertical coherence length $\left(D_{\text {coh } \perp}\right)$ is comparable to layer thickness up to $\sim 680 \mathrm{~nm}$, above which it remains consistently in the 520 to $600 \mathrm{~nm}$ range. This effect is not related to the $\mathrm{x}$-ray penetration depth of the (0002) reflection. We suggest that the variation seen in $D_{\mathrm{coh} \perp}$ is primarily due to the relaxation of elastic strain and character of edge dislocations in the volume of epitaxial layers. Second, we see that the $\omega \mathrm{RC}$ FWHM rises with thickness and is very large for the thick samples (numbered 6-9). The lateral coherence lengths deduced from the $\omega$ RC FWHM shows a maximum $(970 \mathrm{~nm})$ for the $420 \mathrm{~nm}$ thick layer, then decreases considerably $(115-150 \mathrm{~nm})$ for thicker layers. This effect correlates with a noticeable increase of the density of threading screw dislocations in the main part of these layers.

In Fig. 2 we graph the threading screw dislocation density summarized in Table I versus layer thickness. In IIInitride epitaxial structures the density of these dislocations directly correlates with the total amount of generated crystalline defects. The threading dislocation density is high $N_{\mathrm{Th}} \sim(4-7) \times 10^{8} / \mathrm{cm}^{2}$ for thick layers, and low $N_{\mathrm{Th}} \sim 1-2$ $\times 10^{7} / \mathrm{cm}^{2}$ below thickness $0.6 \mu \mathrm{m}$. The minimum value ob- tained is $1.0 \times 10^{7} / \mathrm{cm}^{2}$ at $0.42 \mu \mathrm{m}$ thickness. In Fig. 2 we also graph the shift in the GaN $E_{2}^{2}$ phonon energy as a function of layer thickness for direct comparison with $N_{\mathrm{Th}}$. The $E_{2}^{2}$ phonon shift depends directly on the biaxial epilayer stress $\sigma_{\perp}$. Using Raman-stress factor of $3.4 \pm 0.3 \mathrm{~cm}^{-1} /$ $\mathrm{GPa},{ }^{25}$ we see that the corresponding biaxial tensile stresses in the thin samples are very high, $\sim 1 \mathrm{GPa}$. The stress systematically decreases with thickness above $\sim 0.6 \mu \mathrm{m}$. The 15 to $31 \mu \mathrm{m}$ thick samples are nearly relaxed, with stress $\sim 0.1 \mathrm{GPa}$. We note here that the depth of focus for our micro-Raman instrument is $\sim 2 \mu \mathrm{m}$, so that for the thicker samples we measure stress in the top few microns only. The correlation between stress relaxation from the $E_{2}^{2}$ phonon shift and dislocation density is very convincing in Fig. 2.

Table II shows both in-plane and growth axis strains obtained from XRD measurements. The table also summarizes stress determined from the X-ray results and the related shift in $E_{2}^{2}$ phonon energies. The maximum elastic strains $\left(\varepsilon_{a}\right)$ are seen in the thin $(0.30$ and $0.42 \mu \mathrm{m}) \mathrm{GaN}$ layers, with values of $2.2-3.2 \times 10^{-3}$. This rather large strain is comparable to what we expect from thermal expansion mis-

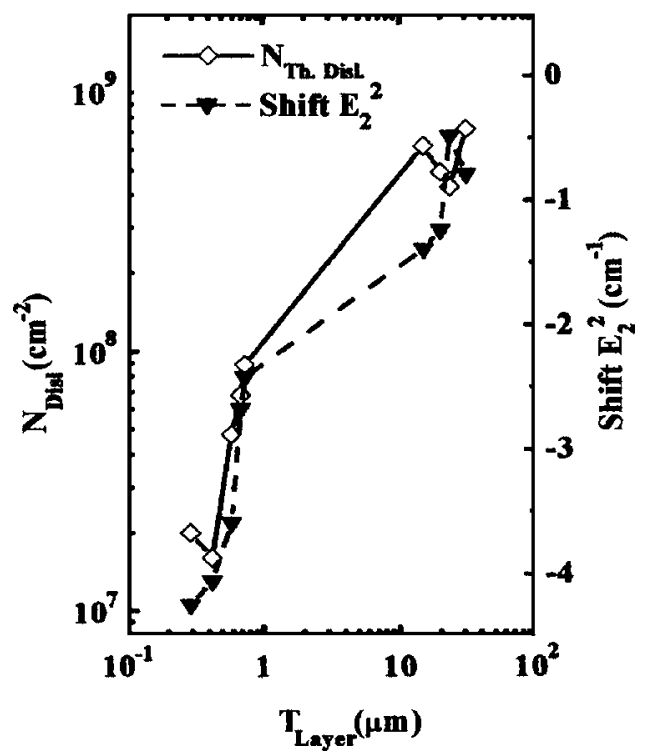

FIG. 2. Correlation of the magnitude of elastic stress values obtained from Raman measurement with the density of threading $\left(N_{\mathrm{Th}}\right)$ dislocations in epitaxial layers, evaluated from the half width of $\omega$ RCs. 
TABLE II. X-ray and Raman strain data.

\begin{tabular}{cccccc}
\hline \hline Sample & $T_{\text {Layer }}(\mu \mathrm{m})$ & $\varepsilon_{c}$ & $\varepsilon_{a}$ & $\begin{array}{c}\sigma_{a}(\mathrm{GPa}) \\
(\mathrm{x} \text { ray })\end{array}$ & $\begin{array}{c}\text { Shift in } E_{2}^{2} \\
\text { phonon }\left(\mathrm{cm}^{-1}\right)\end{array}$ \\
\hline 1 & 0.29 & $-1.55 \times 10^{-3}$ & $2.8 \times 10^{-3}$ & $0.53-1.16$ & -4.25 \\
2 & 0.42 & $-1.35 \times 10^{-3}$ & $2.19 \times 10^{-3}$ & $0.43-0.93$ & -4.06 \\
3 & 0.58 & $-1.35 \times 10^{-3}$ & $2.5 \times 10^{-3}$ & $0.47-1.03$ & -3.59 \\
4 & $\geqslant 0.68$ & $-1.73 \times 10^{-3}$ & $3.14 \times 10^{-3}$ & $0.59-1.30$ & -2.67 \\
5 & $\geqslant 0.73$ & $-1.35 \times 10^{-3}$ & $2.19 \times 10^{-3}$ & $0.43-0.93$ & -2.42 \\
6 & $15 \mu \mathrm{m}$ & $-4.80 \times 10^{-4}$ & $6.3 \times 10^{-4}$ & $0.13-0.28$ & -1.39 \\
7 & $20 \mu \mathrm{m}$ & $-3.85 \times 10^{-4}$ & $7.85 \times 10^{-4}$ & $0.145-0.315$ & -1.24 \\
8 & $25 \mu \mathrm{m}$ & $-3.85 \times 10^{-4}$ & $3.15 \times 10^{-4}$ & $0.075-0.165$ & -0.48 \\
9 & $31 \mu \mathrm{m}$ & $-3.85 \times 10^{-4}$ & $6.9 \times 10^{-4}$ & $0.13-0.285$ & -0.79 \\
\hline \hline
\end{tabular}

match $\sim 2.5 \times 10^{-3}$. In the $15-30 \mu \mathrm{m}$ thick layers, the measured residual elastic strain relaxes to between (3-6) $\times 10^{-4}$.

\section{B. Reciprocal space maps-variation in the epilayer morphology with the depth}

Reciprocal space mapping (RSM) allows us to explore the spatial distribution of elastic strain within the epilayers. The (0002) reflection with shorter extinction length and smaller sensitivity to elastic strain is used to investigate the thin layer samples. For the thicker layers (samples 6-9 in Tables I and II) RSM has been carried out using (0004) and (0006) reflections. The larger extinction lengths and spatial resolutions of these reflections allow us to depth profile the epilayer morphology closer to the $\mathrm{GaN} / \mathrm{SiC}$ interface.

Figures 3(a) and 3(b) show (0002) RSM for thinner layers (1 and 2). Both exhibit a narrow central spot in $q_{z}(2 \theta$ $-\omega)$ direction. Additional interference spots stem from diffraction in the rather perfect domain layers. Small misorientation of crystalline domains leads to elongation of all diffraction spots in lateral $q_{x}(\omega)$ direction. RSM results for the thinnest $(0.29 \mu \mathrm{m})$ epitaxial layer in Fig. 3(a) fully confirm x-ray diffraction data shown in Fig. 1(a). A strong interference pattern, symmetrical in the $q_{z}$ direction, unambiguously shows the lack of relaxation of elastic strain and hence negligible density of edge dislocations in the main part of the layer.

Short elongation of interference spots in the $q_{x}$ direction shows that the main type of crystalline defects in this layer is a coarse accumulation of threading dislocations or dislocation walls, rising from the initial growth, or "accommodation" layer. These extended defects attract mobile point defects, which diffuse in from surrounding areas, thereby increasing the crystalline perfection of these areas. ${ }^{16}$ As a result, the lateral coherent length of the diffracted radiation increases to $0.5-0.6 \mu \mathrm{m}$, apparently corresponding to the average distance between dislocation walls. This suggests that the diffusion length of point defects in the layer is on the order of $0.5 \mu \mathrm{m}$. In contrast to edge dislocations, created in the volume of the epitaxial layer, screw dislocations do not deteriorate the vertical integrity or coherence of the epitaxial layer. Figure 3(b) exhibits a slight asymmetry of the central diffraction spot in the $-q_{z}$ direction. The asymmetry is indicative of the initial stages of relaxation of accumulated elastic strain and appearance of edge dislocations in the volume of the epitaxial layer (thickness $0.42 \mu \mathrm{m}$ ).

The results illustrate the exaggerated problem of lattice mismatch, related with the foreign substrates for the III-N epitaxial layers. Under perfect growth conditions the thin
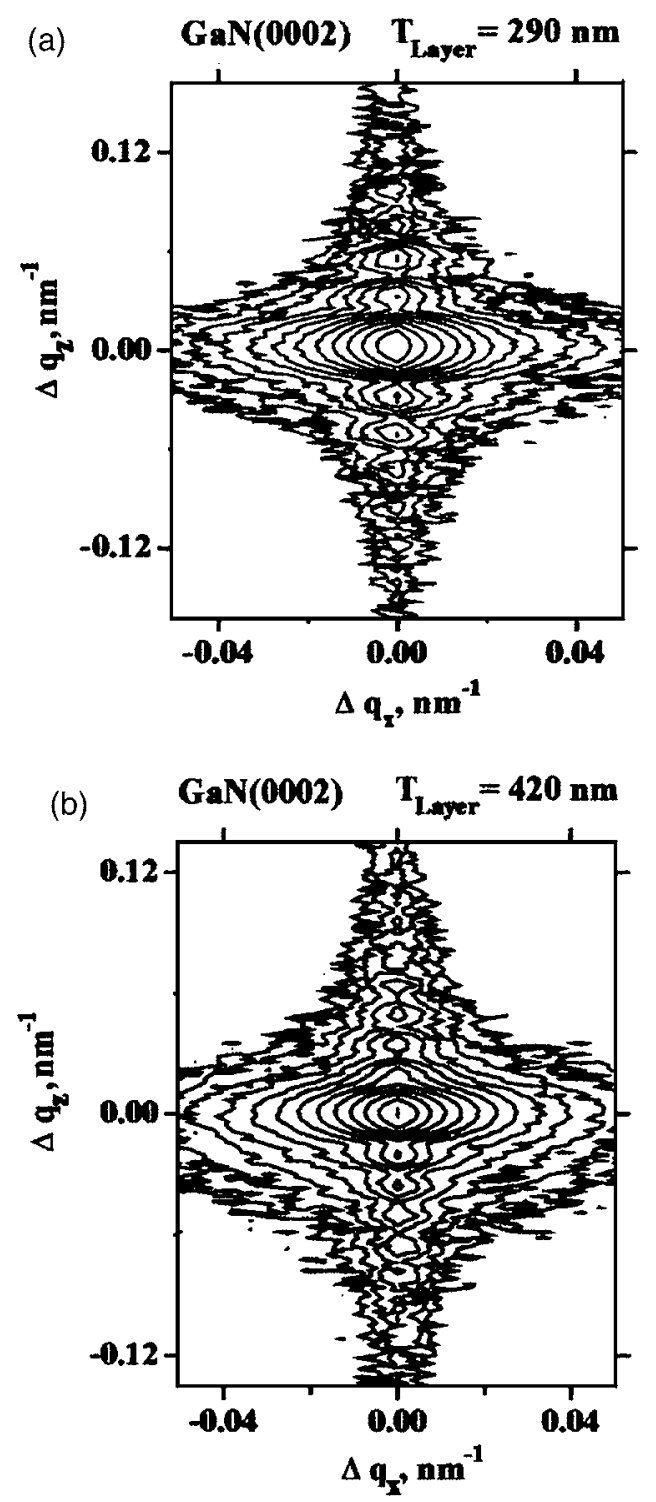

FIG. 3. (a) and (b) Coherent RSM pictures for $290 \mathrm{~nm}$ (sample 1) and $420 \mathrm{~nm}$ (sample 2) thick GaN layers, measured at triple-crystal arrangement in the vicinity of the GaN (0002) reflection. 

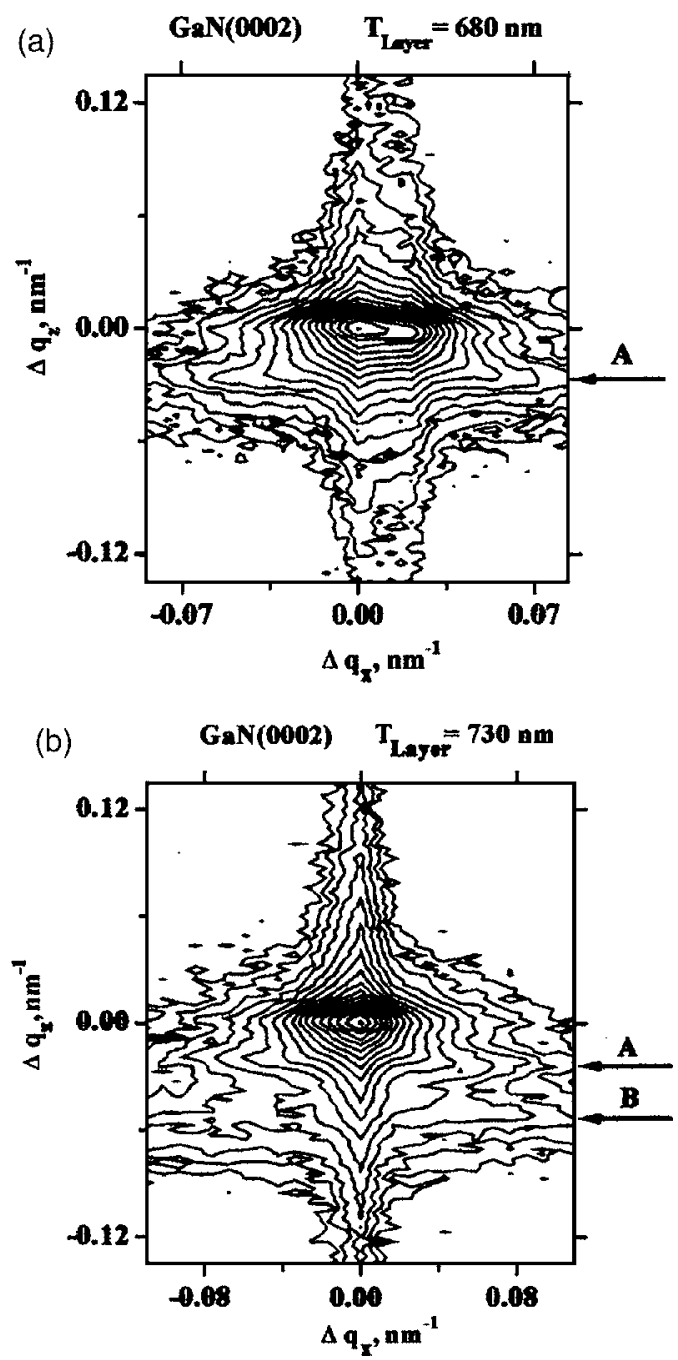

FIG. 4. (a) and (b) Coherent RSM pictures for $680 \mathrm{~nm}$ (sample 4) and $730 \mathrm{~nm}$ (sample 5) thick GaN layers, measured at triple-crystal arrangement in the vicinity of the GaN (0002) reflection. Arrows A and B indicate additional diffracted areas with higher lattice parameter $c$.

initial GaN [or AlN Ref. 15] layer (20-50 nm) accommodates the initial lattice mismatch and significantly diminishes this structure-degrading factor. Following this initial layer, the effects of the initial heterogeneity are residual elastic strain and the presence of extended threading defects.

Further increase of the layer thickness significantly changes the distribution of the diffraction [samples 4 and 5, Figs. 4(a) and 4(b)]. The interference fringes are completely extinguished due to increased heterogeneity in the volume of the layers [Figs. 4(a) and 4(b)]. The lateral broadening of the diffracted spots at $q_{z}$ just below the Bragg maximum indicates an appearance of additional thin sublayers with larger lattice parameter. The appearance of these diffuse spots, shifted in the $-q_{z}$ direction, indicates the next stage of the epitaxial growth process, i.e., the appearance of elastic strain in the volume of the epitaxial layer due to stoichiometry deteriorations, and as a result, their partial relaxation and creation of edge dislocations in the volume of the epitaxial layer at the depth, determined by the diffusion length of point defects. Drastic lateral elongation of the central diffraction spot and additional diffuse spots at $\Delta q_{z}$, denoted as A

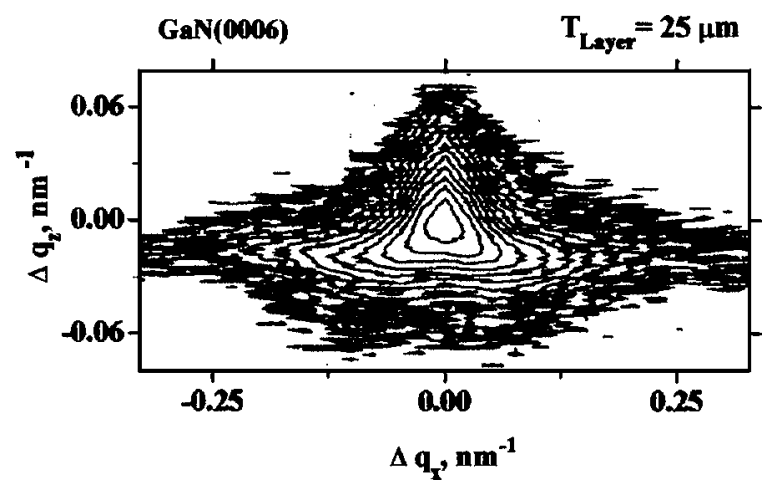

FIG. 5. Coherent RSM picture for $25 \mu \mathrm{m}$ (sample 8) thick GaN layer, measured at triple-crystal arrangement in the vicinity of the GaN (0006) reflection.

and $\mathrm{B}$, arises from a significantly increased density of small threading dislocations (in excess of $10^{9} / \mathrm{cm}^{2}$ ), simultaneously created at these sub-layers. Our analysis of the depth profile relies on x-ray diffraction in absorbent crystals (epitaxial layers) possessing a gradient in lattice parameters, similar to what has been previously described. ${ }^{26,27}$ It also agrees with typical spatial distribution of crystalline defects in GaN epitaxial layers, revealed by TEM. ${ }^{10,13}$

Spatial displacement of diffused spots in negative $q_{z}$ direction, seen in Figs. 4(a) and 4(b), reveals that the lattice parameter $c$ at these sub-layers is larger than in the main part of the layer. Thus, the elastic strain $\varepsilon_{c}$ at this depth is nearly fully relaxed or even slightly positive relative to the main part of the layer. The periodicity seen in the diffused spots in the $q_{z}$ direction [Fig. 4(b)] is reminiscent of XRD from epitaxial layers with a linear lattice parameter gradient, due to the relaxation of elastic strain.

Figure 5 shows the RSM for the GaN (0006) reflection from the $25 \mu \mathrm{m}$ thick layer (sample 8). The greater probe depth associated with the (0006) reflection allows us to obtain a more precise spatial distribution of elastic strain and crystalline defects throughout nearly the entire layer. The RSM pattern shows a pronounced triangular shape: the top convergent part corresponds to the deeper part of the layer, while the wide triangular base corresponds to the fully relaxed part of the layer. Elongation of the diffraction spot along $q_{z}$ shows a gradual relaxation of elastic strain with increased distance from the $\mathrm{GaN} / \mathrm{SiC}$ interface. In the main part of the epilayer these strains range from $\varepsilon_{c}=(-8.0 \pm 1.0)$ $\times 10^{-4}$ up to zero. At the depth of diffusion length of point defects (bottom spot in Fig. 5) the elastic strains are positive, $\varepsilon_{c}(+3.0-+4.0) \times 10^{-4}$, in agreement with the previous discussion in relation to Figs. 4(a) and 4(b).

The diminishing trend seen in the $q_{x}$ width of the diffraction pattern in Fig. 5, as we proceed from the central part of the figure to the top, shows an increase of the size of crystalline defects with increasing distance from the surface of the layer. This is a result of structural transformation of crystalline defects during epitaxial growth. Structural transformation begins together with an appearance of point defects at the growth surface, and evolves consecutively from clusters of point defects, through edge dislocations and threading screw dislocations to domain walls created by screw thread- 
ing dislocations. One main feature of this process is a spatial and temporal dependence of this process on growth conditions due to the generation of point defects and their diffusion away from the growth surface. Smaller crystalline defects, such as clusters of point defects and small dislocations, prevail close to the growth surface, while the majority of point defects diffuse through this region and move deeper into the layer, accumulate, and create extended defects. Therefore, the density of extended defects reaches a maximum at some distance from the surface. This conclusion is consistent with established spatial distributions of threading dislocations, shown by TEM, which show that the density of dislocation walls noticeably diminishes close to the surface of the layers. $5,10,13$

\section{DISCUSSION OF THE POINT DEFECT DEPLETION EFFECT}

The primary experimental results are summarized as follows. An initial accommodation layer, 20 to $50 \mathrm{~nm}$ thick, forms near the GaN/substrate interface with a very high density of dislocations, domain walls, and point defects. This region is immediately followed by $\mathrm{GaN}$ with an unexpectedly low concentration of extended defects. The thickness of this layer extends for the growth conditions used here to $\sim 0.6$ to $0.7 \mu \mathrm{m}$. This layer exhibits residual elastic strain comparable to the thermal strain produced by the substrate. For thicker layers point defects, diffusing from the surface, are captured by extended defects already present in the material, resulting in their growth.

The origin of the initial $\mathrm{GaN}$ accommodation layer with a very high density of defects is well established. ${ }^{10,13}$ Here, the primary source of defects is the extremely high lattice mismatch between the substrate material and the GaN. Beyond the formation of a GaN nucleation layer and recovery of the initial lattice mismatch, defects also arise from the non-ideal stoichiometry and deterioration of the growth surface by threading dislocations, and dislocation walls.

Figure 6 plots the density of threading dislocations together with the expected behavior of other growth factors, as a function of the layer thickness. The initial lattice mismatch, giving rise to heteroepitaxial growth and large dislocation density, rapidly reduces, and the growth transitions to homoepitaxy. This is reflected in the model plot of lattice mismatch at the growth temperature, as a function of layer thickness. This curve addresses the decrease of the density of misfit dislocations in the volume of epitaxial layers, generated due to initial lattice mismatch between substrate and epitaxial layers. For the accommodation layer we use published TEM data for the dislocation density [about $10^{11} / \mathrm{cm}^{2}$ (Refs. 10 and 13)]. For thicker layers (300-500 nm), grown under the lack of volumetric elastic strain (samples 1-3), the influence of this factor becomes negligible. The thermal mismatch increases upon cooling to room temperature, contributing to the stress. ${ }^{25}$

The second plotted factor is stoichiometry of growth conditions. Constant growth stoichiometry is assumed throughout. The average density of the threading dislocation in the thick epitaxial layers (samples 6-9) is used to estimate

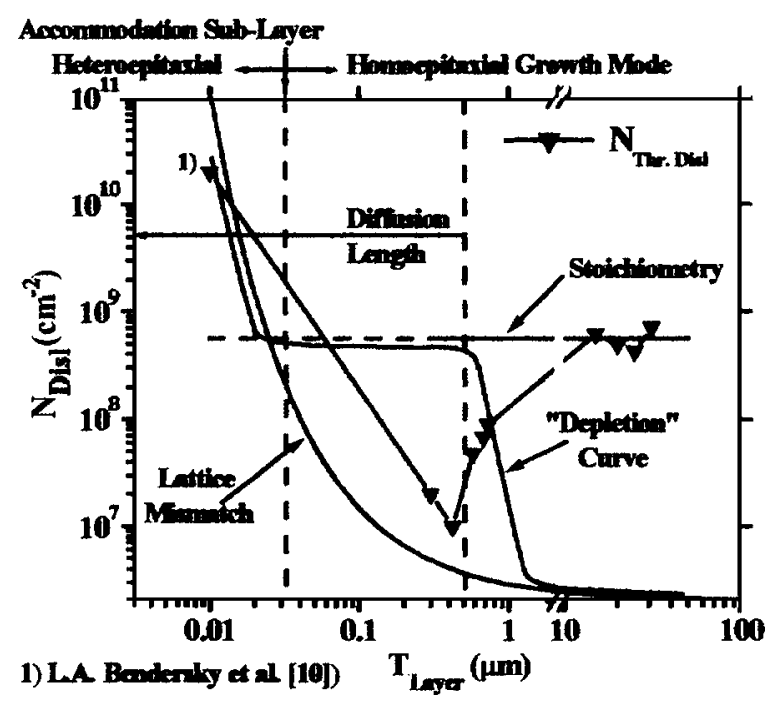

FIG. 6. Density of threading screw dislocations plotted in comparison with other growth factors. For the accommodation layer published TEM data for the dislocation density [about $10^{11} / \mathrm{cm}^{2}$ (Ref. 10)] have been used. Curve labeled "lattice mismatch" is the influence of initial lattice mismatch between substrate and epitaxial structure on the density of edge dislocations. Curve labeled "stoichiometry" shows the influence of growth conditions on crystalline perfection based on the density of threading dislocations at the thick layers. Curve labeled "depletion" illustrates getter ability of threading screw dislocations. Left vertical dashed line depicts thickness of the accommodation layer, while the right one notes estimated diffusion length of vacancy point defects.

the effect of stoichiometry on threading dislocation density, as it represents the best-case situation studied.

The model curve labeled "depletion" evaluates the ability of threading screw dislocations, located in the accommodation layer, to getter point defects diffusing from the growth surface inward. This gettering results in diminished density of threading screw dislocations in the top part of epitaxial layers. The gettering should be maximum in the accommodation layer and diminished in the near-surface part of the layer. Gettering should be negligible when the thickness of the epitaxial layer exceeds the diffusion length of the point defects generated at the surface. We estimate the diffusion length to be $\sim 0.5 \mu \mathrm{m}$ based on the thickness of the layer with the minimal density of threading screw dislocations.

The observed distribution of dislocation densities could be considered in terms of a diffusion model in which point defects diffuse from the surface inward and are gettered by preexisting extended defects. Once a point defect is captured it becomes immobile, resulting in anisotropic diffusivity. For thin layers we consider a straightforward model, based on Fick's laws. Two layers describe the near-surface region, with point defect diffusivity $D_{1}$ corresponding to better crystal quality, and a second layer, with high extended defect concentration and diffusivity $D_{2}$

$$
D(x)=\left\{\begin{array}{ll}
D_{1}, & 0 \leqslant x \leqslant x_{1} \\
D_{2}, & x \geqslant x_{1}
\end{array},\right.
$$

where $x_{1}$ is the thickness of the topmost layer. In this model $x$ is in the reference frame of the growing surface. When $D_{1}$ and $D_{2}$ are not equal, we have an anisotropic diffusion along the $x$ direction. With $D_{2} \ll D_{1}$, this model adequately depicts 
a surface region with low density of point defects, residing between the dashed lines in Fig. 6, and a deeper region with much higher density. Physically, the majority of point defects locked in by the low $D_{2}$ are transformed into extended defects, and the effectiveness of this mechanism depends on their density. Because we observe the near-surface region to maintain a nearly constant thickness of $\sim 0.5 \mu \mathrm{m}$ with growth time, we attribute this to a balance between several factors, primarily the point defect generation rate, i.e., stoichiometry of growth conditions, the inward diffusion, and density of already existing crystalline defects. For layers thinner than $0.5 \mu \mathrm{m}$, the accommodation layer functions to getter point defects. When the total $\mathrm{GaN}$ layer thickness is greater than this value, the intermediate or main layer develops and the accommodation layer becomes stagnant. In other words, when the thickness of the layer exceeds diffusion length, the importance of depletion is reduced.

\section{SUMMARY}

High resolution x-ray diffraction and Raman spectroscopy have been used to examine the relaxation of elastic strain in $\mathrm{GaN}$ epitaxial layers grown on $\mathrm{SiC}$ substrates. We examine a wide thickness range $(0.29$ to $31 \mu \mathrm{m})$ and use several Bragg reflections to obtain depth profiles of elastic strain and evaluate crystalline perfection. Our results suggest a layered morphology. A thin initial GaN layer $(25-50 \mathrm{~nm})$ accommodates the lattice mismatch between substrate and epitaxial layer. This layer exhibits high density of extended defects, consistent with TEM investigations, arising from the lattice mismatch between $\mathrm{GaN}$ and $\mathrm{SiC}$ at the growth temperature. In this accommodation layer the growth changes from hetero- to homo-epitaxy. Subsequent layers, for samples grown with the total thickness below $0.6 \mu \mathrm{m}$, have significantly lower dislocation density and exhibit stresses consistent with thermal expansion mismatch between the substrate and epilayer. However, if thicker layers are grown, an additional intermediate region is observed, in which the extended defect density is between that of the initial accommodation layer and that of the near-surface material.

We propose a diffusion model based on two layers. The first layer corresponds to the growing layer, through which point defects, generated at the growth surface, readily diffuse. The layer beneath traps diffusing point defects by incorporating them into existing and newly extended defects. This results in anisotropy of the point defect diffusivity. Under the growth conditions used here, the point defects in thin $(<1 \mu \mathrm{m})$ epitaxial layers have diffusion lengths comparable to the layer thickness, and thus diffuse to the extended defects in the accommodation layer where they are trapped. This process allows a high-quality near-surface layer to form. For thicker layers, point defects do not reach the accommodation layer, and are instead incorporated into extended defects. The main factors in this model are stoichiometry of growth conditions, density of threading screw dislocations in the accommodation layer, and the inward diffusion of point defects.

\section{ACKNOWLEDGMENTS}

The authors thank V. Dmitriev for interest in this study and fruitful discussions. Work at Texas Tech University was partially supported by the National Science Foundation (ECS-0323640).

${ }^{1}$ H. Morkoc, S. Strite, G. B. Gao, M. E. Lin, B. Sverdlov, and M. Burns, J. Appl. Phys. 76, 1363 (1994).

${ }^{2}$ S. C. Jain, M. Willander, J. Narayan, and R. Van Overstraeten, J. Appl. Phys. 87, 965 (2000).

${ }^{3}$ S. J. Pearton, J. C. Zolper, R. J. Shul, and F. Ren, J. Appl. Phys. 86, 1 (1999).

${ }^{4}$ F. A. Ponce, B. S. Krusor, J. S. Major, Jr., W. E. Plano, and D. F. Welch, Appl. Phys. Lett. 67, 410 (1995).

${ }^{5}$ S. A. Nikishin, N. N. Faleev, V. G. Antipov, S. Francoeur, L. Grave de Peralta, G. A. Seryogin, H. Temkin, T. Prokofyeva, M. Holtz, and S. N. G. Chu, Appl. Phys. Lett. 75, 2073 (1999); (see also J. P. Zhang, H. M. Wang, M. E. Gaevski, C. Q. Chen, Q. Fareed, J. W. Yang, G. Simin, and M. A. Khan, ibid. 80(19), 3542 (2002); H. M. Wang, J. P. Zhang, C. Q. Chen, Q. Fareed, J. W. Yang, and M. A. Khan, ibid. 81, 604 (2002)).

${ }^{6}$ F. A. Ponce, D. P. Bour, W. Götz, N. M. Jonson, H. I. Helava, I. Grzegory, and S. Porowski, Appl. Phys. Lett. 68, 917 (1996).

${ }^{7}$ N. N. Faleev, A. Yu. Egorov, A. E. Zhukov, A. R. Kovsh, S. S. Mikhrin, V. M. Ustinov, K. M. Pavlov, V. I. Punegov, M. Tabuchi, and Y. Takeda, Semiconductors 33, 1229 (1999); N. N. Faleev, Yu. G. Musikhin, A. A. Suvorova, A. Yu. Egorov, A. E. Zhukov, A. R. Kovsh, V. M. Ustinov, M. Tabuchi, and Y. Takeda, ibid. 35, 969 (2001).

${ }^{8}$ J. Tersoff and F. K. LeGoues, Phys. Rev. Lett. 72, 3570 (1994).

${ }^{9}$ V. G. Gruzdov, A. O. Kosogov, and N. N. Faleev, Tech. Phys. Lett. 20, 561 (1994).

${ }^{10}$ L. A. Bendersky, D. V. Tsevtkov, and Yu. V. Melnik, J. Appl. Phys. 94, 1676 (2003).

${ }^{11}$ L. E. Shilkrot, D. J. Srolovitz, and J. Tersoff, Phys. Rev. B 62, 8397 (2000) (and references therein).

${ }^{12}$ A. A. Chernov, Modern Crystallography III, Crystal Growth (SpringerVerlag, Berlin, Heidelberg, 1984), Chaps. 1, 2.

${ }^{13}$ X. J. Ning, F. R. Chien, P. Pirouz, J. W. Yang, and M. A. Khan, J. Mater. Res. 11, 580 (1996); X. N. Wu, L. M. Brown, D. Kapolnek, S. Keller, B. Keller, S. P. DenBaars, and J. S. Speck, J. Appl. Phys. 80, 3228 (1996).

${ }^{14}$ T. S. Zheleva, O. H. Nam, M. D. Bremser, and R. F. Davis, Appl. Phys. Lett. 71, 2472 (1997)

${ }^{15} \mathrm{~N}$. Faleev (private communication).

${ }^{16}$ A. Kazimirov, N. N. Faleev, H. Temkin, M. J. Bedzyk, V. Dmitriev, and Yu. Melnik, J. Appl. Phys. 89, 6092 (2001).

${ }^{17}$ H. Marchand, L. Zhao, N. Zhang, B. Moran, R. Coffie, U. K. Mishra, J. S. Speck, S. P. DenBaars, and J. A. Freitas, J. Appl. Phys. 89, 7846 (2001), and references therein.

${ }^{18}$ Yu. V. Melnik, I. P. Nikitina, A. S. Zubrilov, A. A. Sitnikova, Yu. G. Musikhin, and V. A. Dmitriev, Inst. Phys. Conf. Ser. 142, 863 (1996).

${ }^{19}$ I. Nikitina, G. Mosina, Yu. Melnik, A. Nikolaev, and K. Vassilevski, Mater. Sci. Eng., B 61-62, 325 (1999).

${ }^{20}$ P. F. Fewster and N. L. Andrew, J. Appl. Crystallogr. 28, 451 (1995).

${ }^{21}$ C. Kisielowski, J. Krüger, S. Ruvimov, T. Suski, J. W. Ager III, E. Jones, Z. Liliental-Weber, M. Rubin, E. R. Weber, M. D. Bremser, and R. F. Davis, Phys. Rev. B 54, 17745 (1996).

${ }^{22}$ M. Holtz, M. Seon, T. Prokofyeva, H. Temkin, R. Singh, F. P. Dabkowski, and T. D. Moustakas, Appl. Phys. Lett. 75, 1757 (1999).

${ }^{23}$ K. G. Fertitta, A. L. Holmes, F. J. Ciuba, R. D. Dupuis, and F. A. Ponce, J. Electron. Mater. 24, 257 (1995).

${ }^{24}$ J. P. Zhang, M. A. Khan, W. H. Sun, H. M. Wang, C. Q. Chen, Q. Fareed, E. Kuokstis, and J. W. Yang, Appl. Phys. Lett. 81, 4392 (2002).

${ }^{25}$ I. Ahmad, M. Holtz, N. Faleev, and H. Temkin, J. Appl. Phys. 95, 1692 (2004).

${ }^{26}$ V. I. Punegov and N. N. Faleev, Phys. Solid State 38, 143 (1996).

${ }^{27}$ V. I. Punegov, K. M. Pavlov, S. G. Podorov, and N. N. Faleev, Phys. Solid State 38, 148 (1996). 